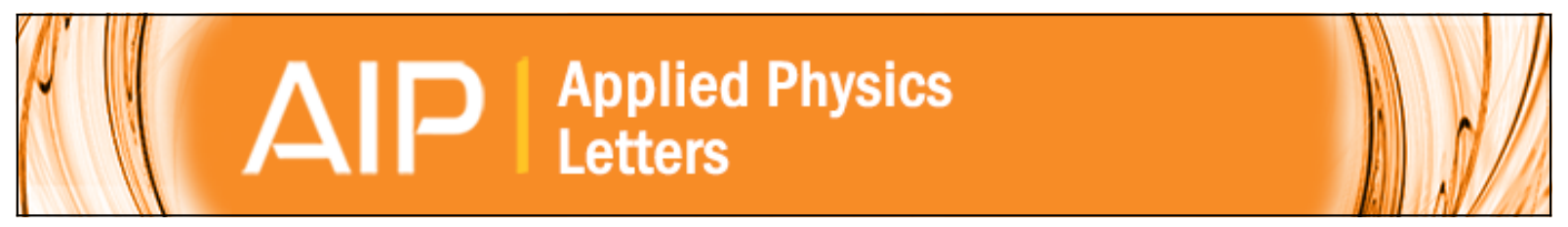

Optical cavity with a double-layered cholesteric liquid crystal mirror and its prospective application to solid state laser

Soon Moon Jeong, Koji Sonoyama, Yoichi Takanishi, Ken Ishikawa, Hideo Takezoe, Suzushi Nishimura, Goro

Suzaki, and Myoung Hoon Song

Citation: Applied Physics Letters 89, 241116 (2006); doi: 10.1063/1.2404937

View online: http://dx.doi.org/10.1063/1.2404937

View Table of Contents: http://scitation.aip.org/content/aip/journal/apl/89/24?ver=pdfcov

Published by the AIP Publishing

Articles you may be interested in

Mechanically tunable microlasers based on highly viscous chiral liquid crystals

Appl. Phys. Lett. 99, 233302 (2011); 10.1063/1.3665943

Tunable semiconductor vertical-cavity surface-emitting laser with an intracavity liquid crystal layer Appl. Phys. Lett. 98, 161105 (2011); 10.1063/1.3569591

Defect mode lasing from a double-layered dye-doped polymeric cholesteric liquid crystal films with a thin rubbed defect layer

Appl. Phys. Lett. 90, 261108 (2007); 10.1063/1.2752017

Doubling the optical efficiency of a chiral liquid crystal laser using a reflector

Appl. Phys. Lett. 87, 231107 (2005); 10.1063/1.2138353

Cholesteric liquid crystal laser with wide tuning capability

Appl. Phys. Lett. 86, 161120 (2005); 10.1063/1.1897439

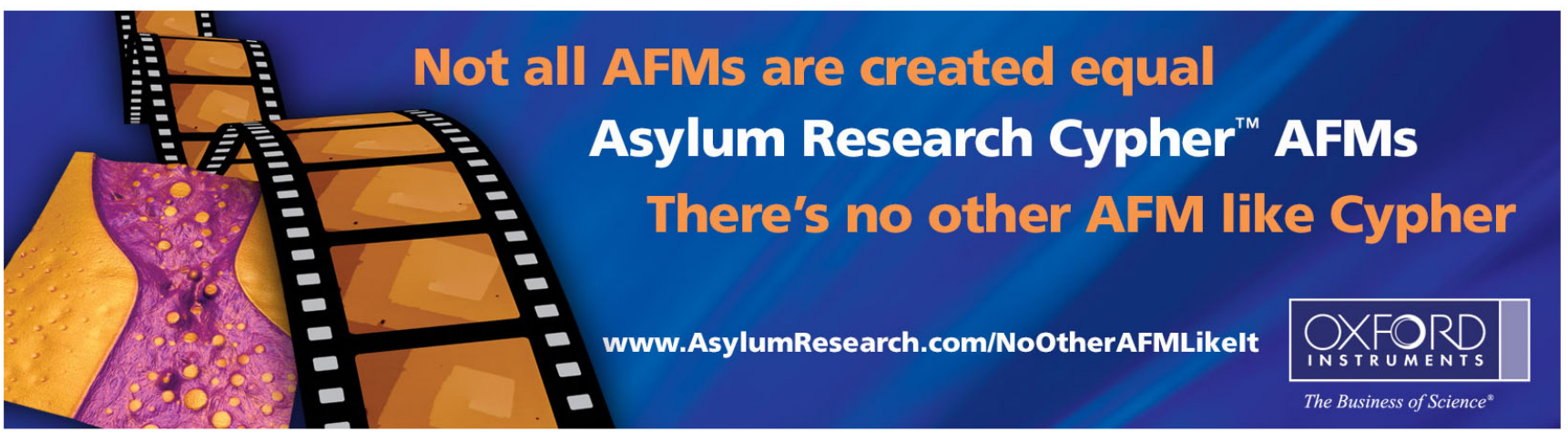




\title{
Optical cavity with a double-layered cholesteric liquid crystal mirror and its prospective application to solid state laser
}

\author{
Soon Moon Jeong, Koji Sonoyama, Yoichi Takanishi, Ken Ishikawa, and Hideo Takezoe ${ }^{\text {a) }}$ \\ Department of Organic and Polymeric Materials, Tokyo Institute of Technology, 2-12-1, O-okayama, \\ Meguro-ku, Tokyo 152-8552, Japan \\ Suzushi Nishimura and Goro Suzaki \\ Nippon Oil Corporation, Central Technical Research Laboratory, 8 Chidori-cho, Naka-ku, \\ Yokohama 231-0815, Japan \\ Myoung Hoon Song \\ Department of Chemical and Biomolecular Engineering, Korea Advanced Institute of Science \\ and Technology, Daejeon 305-701, Korea
}

(Received 11 August 2006; accepted 31 October 2006; published online 14 December 2006)

\begin{abstract}
The authors have fabricated an optical cavity with silver $(\mathrm{Ag})$ and double-layered cholesteric liquid crystal (CLC) mirrors facing each other. This CLC mirror consists of left-handed CLC and right-handed CLC films for high light reflection irrespective of polarization states. A single-mode lasing was observed in dye-doped CLC sandwiched between Ag and double-layered CLC mirrors. The authors also fabricated a flexible solid state device with a spin-coated dye molecular film sandwiched between Ag and double-layered CLC mirrors. Amplified spontaneous emission was observed from the solid state device, suggesting a possible structure for a flexible and tunable solid state laser. (C) 2006 American Institute of Physics. [DOI: 10.1063/1.2404937]
\end{abstract}

Cholesteric liquid crystals (CLCs) are one of the most interesting one-dimensional photonic crystals because the periodic helical structure is spontaneously formed and it exhibits an unusual optical property of selective reflection; circularly polarized light (CPL) with the same handedness of CLC helix is totally reflected when it is incident along the helical axis of CLC and the wavelength $\lambda$ is the same as the optical pitch of the CLC. For sufficiently thick films, the reflectance of CPL with the same sign of rotation as the CLC helix is $100 \%$ within a frequency range corresponding to the photonic band gap (PBG). ${ }^{1,2}$ Dowling et al. predicted that lasing should occur at the edge of a photonic stop band. ${ }^{3}$ Thus, CLCs have been studied for various optical phenomena, particularly for optical lasing, because light within the PBG is confined and amplified at the PBG edge where the photon group velocity approaches zero. ${ }^{4-12}$ However, due to its own optical property of selective reflection, a cavity cannot be formed when the metal mirror is adopted as an opposite mirror to a CLC film because of the phase change due to the reflection. Namely, circularly polarized light reflected by the CLC film changes its polarization to the opposite sense of rotation during reflection at the metal surface, resulting in no reflection at the CLC film when the light is reflected back to the CLC film. ${ }^{13,14}$ This phenomenon makes it difficult to apply CLCs to organic solid state laser with the same structure as organic light-emitting diodes which inevitably require a metal electrode for efficient charge injection.

In this work, we fabricated an optical cavity formed by metal and CLCs for the first time. To make CLCs totally reflect both left CPL (L-CPL) and right CPL (R-CPL), we fabricated a double-layered CLC mirror consisting of CLC films with L- and R-handed helices. The double-layered CLC film is expected to be used for electrically pumped organic

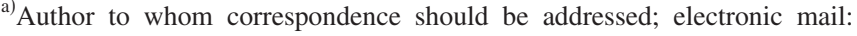
htakezoe@o.cc.titech.ac.jp solid state lasers, as we have already shown the cavity effect in an organic light-emitting diode (OLED) device. ${ }^{15}$

In order to maximize the light reflection irrespective of polarization states, we combined a left-handed CLC (LCLC) film and a right-handed CLC (R-CLC) film as shown in Fig. 1(a). One side of both 2 - $\mu$ m-thick CLC layers is covered with protective layers, which are highly transparent isotropic polymeric films of $80 \mu \mathrm{m}$ in thickness. When light

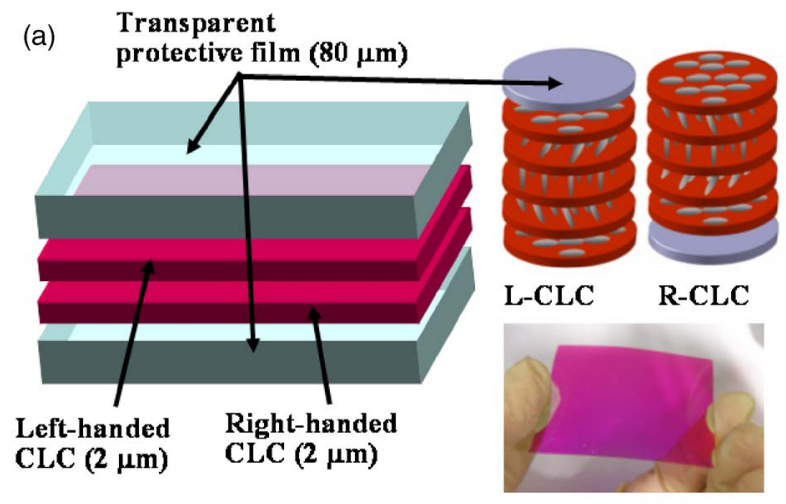

(b)

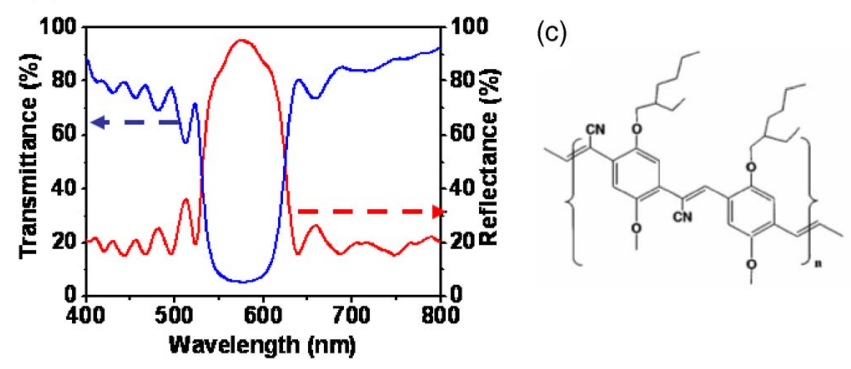

FIG. 1. (Color online) (a) Schematic diagram of double-layered CLC mirror and its photograph. (b) Transmittance and reflectance spectra of the doublelayered (L- and R-helix) CLC mirror. (c) Molecular structure of the dye used. 

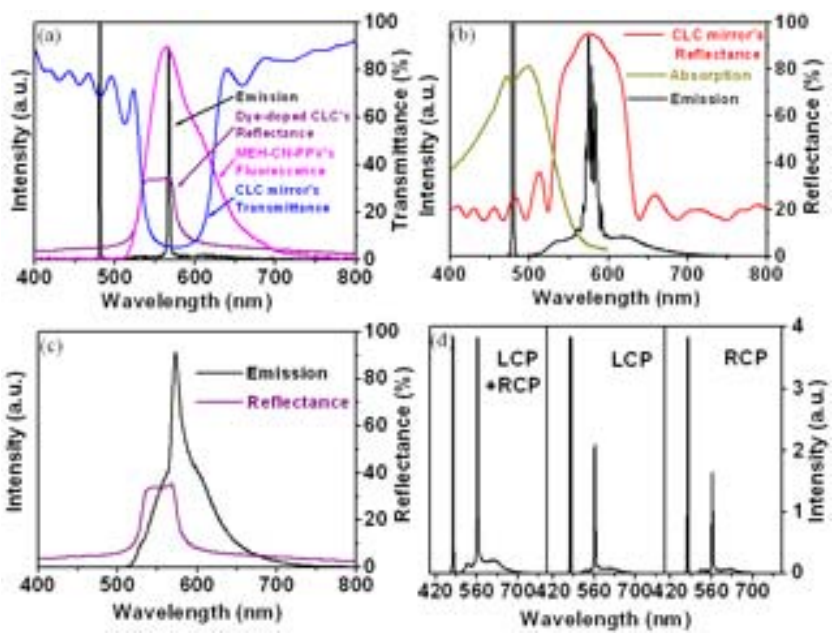

FIG. 2. (Color online) (a) Emission spectrum from dye-doped CLC with Ag and double-layered CLC mirrors. Transmittance spectrum of a doublelayered CLC, reflectance spectrum of a dye-doped CLC, and fluorescence spectrum of MEH-CN-PPV are also shown. (b) Emission spectrum from dye-doped NLC devices with Ag and double-layered CLC mirrors. Multiple lasing emission is observed. (c) Emission spectra from a dye-doped CLC device without mirrors. ASE is observed. (d) Polarization characteristics of the dye-doped CLC device with Ag and double-layered CLC mirrors.

is incident on the double-layered CLC film, L-CPL is reflected by the L-CLC film and R-CPL passes through. The transmitted R-CPL is reflected by the R-CLC film. The transmittance and reflectance spectra for normally incident unpolarized light for a double-layered CLC mirror are shown in Fig. 1(b). Transmittance and reflectance spectra were measured using a microscope spectrometer (TFM-120AFT-PC, ORC). The total reflectance can be maximized over $90 \%$ by combining L-CLC and R-CLC films with the width of stop band of $525-640 \mathrm{~nm}$.

Dye-doped L-CLC was used as an active layer. L-CLC consists of a nematic liquid crystal (NLC) (ZLI2293, Merck, $77.7 \mathrm{wt} \%$ ) and chiral dopant (MLC6247, Merck, $22.3 \mathrm{wt} \%)$. The dye used was poly[2-methoxy-5(2'-ethylhexyloxy)-1,4-(1-cyanovinylene)phenylene] (MEHCN-PPV; obtained from H. W. Sands Corp.), as shown in Fig. 1(c), and the concentration was 2 wt $\%$. The sample cell was made of glass substrates coated with a $\mathrm{Ag}$ layer of $200 \mathrm{~nm}$ thick and a double-layered CLC mirror, which were sustained by a $12-\mu \mathrm{m}$-thick spacer. The sample was introduced into an empty cell using capillary action. For fluorescence and lasing emission measurements, a pulsed laser beam from an optical parametric oscillator (Surelite OPO; HOYA Continuum) pumped by third-harmonic light from a neodymium doped yttrium aluminum garnet laser was used as an optical pumping source. For efficient optical pumping, we adopted $480 \mathrm{~nm}$, corresponding to the absorption peak, as a wavelength of input pulsed laser. The pumping laser beam was focused on the sample surface at oblique incidence (about $30^{\circ}$ ) and the emission from the sample cell was collected by a lens along the direction parallel to the helical axis (normal to the substrate) and then detected by a multichannel spectrometer (USB 2000: Ocean Optics, Inc.).

Figure 2(a) shows a lasing emission spectrum from a dye-doped L-CLC sandwiched between Ag and doublelayered CLC mirrors. Single-mode lasing was clearly observed, being attributed to photonic band edge of L-CLC at which photon group velocity approaches zero. For the threshold value, we plotted the dependence of the laser emission intensity on input pump energy (not shown). It was obviously shown that the differential function of the intensity is discontinuous. We define the break point of the differential function as a threshold energy and it was found to be $18 \mu \mathrm{J}$ pulse $\mathrm{p}^{-1}$.

To confirm the effect of optical cavity, we fabricated two reference cells, (1) dye-doped NLC with mirrors and (2) dyedoped CLC devices without mirrors. We carried out the lasing experiments using the two reference cells. In dye-doped NLC sandwiched between Ag and double-layered CLC mirrors, multiple lasing peaks were clearly observed, as shown in Fig. 2(b), although the lasing threshold is much higher $\left(65 \mu \mathrm{J}\right.$ pulse $^{-1}$ ) compared with that in L-CLC between $\mathrm{Ag}$ and double-layered CLC mirrors. In dye-doped CLC without mirrors, amplified spontaneous emission (ASE) with full width at half maximum (FWHM) of $\sim 10 \mathrm{~nm}$ was observed, as shown in Fig. 2(c). These experiments clearly assure the existence of a cavity made of metal and double-layered CLC mirrors. The lasing condition was improved, i.e., lower threshold and narrower FWHM, by introducing the cavity structure consisting of metal and CLC mirrors. ${ }^{16}$ Enhanced lasing emission has been reported using similar structures at surfaces. ${ }^{17,18}$

It is well known that lasing emission from dye-doped CLC is circularly polarized with the same handedness as that of the CLC helix. ${ }^{4}$ What is the polarization state of the lasing emission in the present case, where dye-doped L-CLC is sandwiched by Ag and double-layered (R-and L-) CLC mirrors? For the polarization characteristic measurements, polarizers were placed between the sample cell and the spectrometer. As shown in Fig. 2(d), the lasing emission was not circularly polarized, i.e., no priority of R- and L-CPL was observed despite the use of the L-handed dye-doped CLC. The following two factors are responsible for the polarization characteristics: (1) The polarization is altered during reflection at the metal surface, and (2) the double-layered CLC film consists of R- and L-CLC films, and thus behaves as a total reflection mirror. Thus, it is reasonable that the difference of the amount of L-CPL and R-CPL is not large irrespective of the polarization state first formed.

To further investigate the cavity characteristics, the spontaneous emission spectra were measured in a thin MEHCN-PPV film sandwiched between Ag and double-layered CLC mirrors, as shown in the inset of Fig. 3(b). MEH-CNPPV was spin coated on a double-layered CLC mirror up to a thickness of $450 \mathrm{~nm}$ and then $\mathrm{Ag}$ was deposited on the polymer film by thermal evaporation up to $200 \mathrm{~nm}$ thickness. Emission characteristics and threshold behavior were measured by optically pumping the device. ASE was observed at the band edge of the double-layered CLC mirror, as shown in Figs. 3(a) and 3(b). This FWHM $(\sim 10 \mathrm{~nm})$ is somewhat broader than that of thin films of small-molecular-weight organic semiconductors which are deposited by thermal evaporation in vacuum. ${ }^{19-22}$ In contrast to small-molecule-based organic vertical-cavity surface-emitting laser emission, it was reported that the emission spectrum has a broad bandwidth even above threshold in spin-coated polymer films. ${ }^{23,24}$ The mechanism of line narrowing was a subject of intense argument, but most researchers now agree that this narrow light is ASE. ${ }^{25}$ The broad bandwidth was attributed to low $Q$ factor $^{26}$ and nonuniformity of the cavity length. ${ }^{27}$ The present device was for optical pumping. It should be noted, 

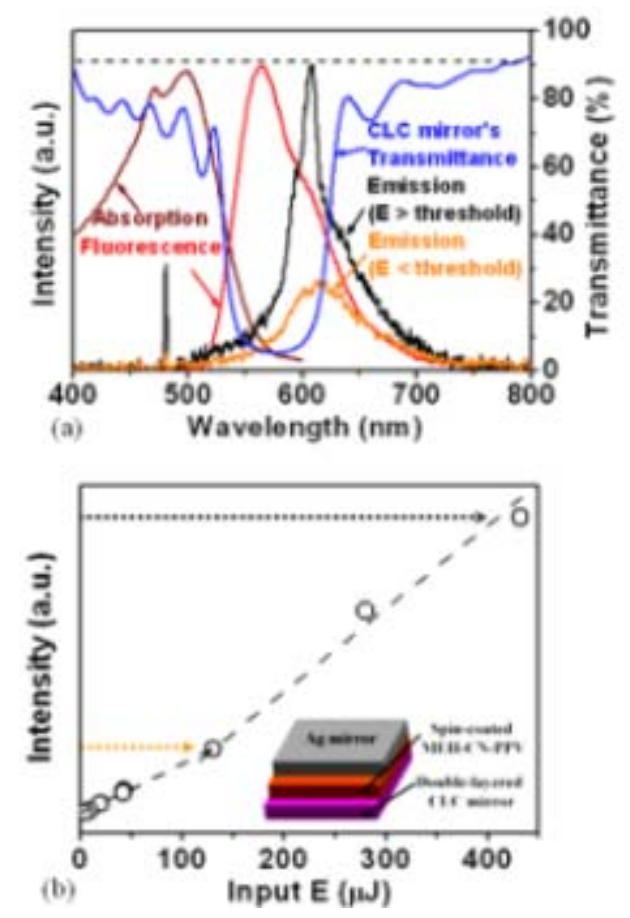

FIG. 3. (Color online) (a) Absorption (brown) and fluorescence (red) spectra of the dye used. For a solid state device [see inset in (b)], i.e., a doublelayered CLC film on which a dye molecular film was spin coated and then $\mathrm{Ag}$ was vacuum evaporated, emission spectra (orange and black) are influenced by the reflection spectrum of the double-layered CLC (blue curve). ASE (black) is observed when input power is above threshold. (b) Threshold behavior of the solid state device shown in the inset.

however, that this structure can be applied to electrically pumped device by depositing an additional metal mirror for electron injection.

Although lasing was not achieved in this experiment, we have confirmed the possibility that the optical cavity used in this experiment can be applied to solid state laser devices. There are several attractive characteristics in a metal/doublelayered CLC cavity structure, tunability and flexibility. Diaz-Garcia et $a{ }^{24}$ have found that single-mode operation is only realized when the peak is located within $\sim 5 \mathrm{~nm}$ of the gain maximum in a Poly(2-butyl, 5-(2'-ethyl-hexyl)-1,4phenylene vinylene) (BuEH-PPV) microcavity. This means that there is no controllable factor determining the singlemode peak position because the optical cavity thickness must be adjusted to the gain maximum. To change the peak position, an emissive polymer with gain maximum corresponding to peak position must be required. In contrast to the microcavity structure, our structure has various factors which govern the wavelength of stimulated light because the stimulated emission occurs at the band edge of the double-layered CLC mirror and the helical pitch (band position) can be controlled by the amount of doped chiral unit or by stretching the CLC film. ${ }^{28}$ Therefore a wide range of peak position is possible by combination of emissive material and doublelayered CLC's band position. Moreover, the use of a mechanically flexible substrate could be an additional advantage for laser technology and might lead to completely novel applications in instrumentation and display technology, which relies on large-area emitters and geometries not accessible by conventional inorganic laser materials.

In summary, we have fabricated the optical cavity with a metal mirror and a doubie-layered $\mathrm{CLC}$ mirror with high reflectance irrespective of polarization states. A single-mode lasing was observed in dye-doped L-CLC sandwiched between metal and double-layered CLC mirrors owing to the confinement of spontaneous emission. We also fabricated a flexible solid state device with spin-coated MEH-CN-PPV and demonstrated amplified spontaneous emission by optical pumping. This device is applicable for flexible and tunable electrically pumped OLED laser, if fundamental problems are solved in the future.

This work is partly supported by a Grant-in-Aid for Exploratory Research (16656023) by the Ministry of Education, Science, Sports and Culture and a New Energy Technology Development Organization grant (Project ID 04A24509). The authors acknowledge Merck Japan Ltd. for supplying NLC (ZLI2293) and the chiral dopant (MLC6247).

${ }^{1}$ E. Yablonovitch, Phys. Rev. Lett. 58, 2059 (1987)

${ }^{2}$ S. John, Phys. Rev. Lett. 58, 2486 (1987).

${ }^{3}$ J. P. Dowling, M. Scalora, M. J. Bloemer, and C. M. Bowden, J. Appl. Phys. 75, 1896 (1994).

${ }^{4}$ V. I. Kopp, B. Fan, H. K. M. Vithana, and A. Z. Genack, Opt. Lett. 23, 1707 (1998).

${ }^{5}$ A. Munoz, P. Palffy-Muhoray, and B. Taheri, Opt. Lett. 26, 804 (2001).

${ }^{6}$ K.-C. Shin, F. Araoka, B. Park, Y. Takanishi, K. Ishikawa, Z. Zhu, T. M. Swager, and H. Takezoe, Jpn. J. Appl. Phys., Part 1 43, 632 (2004).

${ }^{7}$ F. Araoka, K.-C. Shin, Y. Takanishi, Z. Zhu, T. M. Swager, K. Ishikawa, and H. Takezoe, J. Appl. Phys. 94, 279 (2003).

${ }^{8}$ J. Schmidtke, W. Stille, H. Finkelmann, and S. T. Kim, Adv. Mater. (Weinheim, Ger.) 14, 746 (2002).

${ }^{9}$ H. Finkelmann, S. T. Kim, A. Munoz, P. Palffy-Muhoray, and B. Taheri, Adv. Mater. (Weinheim, Ger.) 13, 1069 (2002).

${ }^{10}$ T. Matsui, R. Ozaki, K. Funamoto, M. Ozaki, and K. Yoshino, Appl. Phys. Lett. 81, 3741 (2002).

${ }^{11}$ Y. Matsuhisa, R. Ozaki, M. Ozaki, and K. Yoshino, Jpn. J. Appl. Phys., Part 2 44, L629 (2005).

${ }^{12}$ M. H. Song, N. Y. Ha, K. Amemiya, B. Park, Y. Takanishi, K. Ishikawa, J. W. Wu, S. Nishimura, T. Toyooka, and H. Takezoe, Adv. Mater. (Weinheim, Ger.) 18, 193 (2006).

${ }^{13}$ M. Grell, M. Oda, K. S. Whitehead, A. Asimakis, D. Neher, and D. D. C. Bradley, Adv. Mater. (Weinheim, Ger.) 13, 577 (2001).

${ }^{14}$ V. Vaenkatesan, R. T. Wegh, J. Teunissen, J. Lub, C. W. M. Bastiaansen, and D. J. Broer, Adv. Funct. Mater. 15, 138 (2005).

${ }^{15}$ S. M. Jeong, Y. Takanishi, K. Ishikawa, and H. Takezoe, Jpn. J. Appl. Phys., Part 2 45, L737 (2006).

${ }^{16}$ K. Amemiya, M. H. Song, Y. Takanishi, K. Ishikawa, S. Nishimura, T. Toyooka, and H. Takezoe, Jpn. J. Appl. Phys., Part 1 44, 7966 (2005).

${ }^{17}$ K. Amemiya, T. Nagata, M. H. Song, Y. Takanishi, K. Ishikawa, S. Nishimura, T. Toyooka, and H. Takezoe, Jpn. J. Appl. Phys., Part 1 44, 3748 (2005).

${ }^{18}$ Y. Zhou, Y. Huang, A. Rapaport, M. Bass, and S.-T. Wu, Appl. Phys. Lett. 87, 231107 (2005).

${ }^{19}$ V. G. Kozlov, V. Bulovic, P. E. Burrows, M. Baldo, V. B. Khalfin, G. Parthasarathy, and S. R. Forrest, J. Appl. Phys. 84, 4096 (1998).

${ }^{20}$ V. Bulovic, V. G. Kozolov, V. B. Khalfin, and S. R. Forrest, Science 279, 553 (1998).

${ }^{21}$ A. Dodabalapur, M. Berggren, R. E. Slusher, Z. Bao, A. Timko, P. Schiortino, El. Laskowski, H. E. Katz, and O. Nalamasu, IEEE J. Sel. Top. Quantum Electron. 4, 67 (1998).

${ }^{22}$ M. D. Mcgehee, M. A. DiazGarcia, F. Hide, R. Gupta, E. K. Miller, D. Moses, and A. J. Heeger, Appl. Phys. Lett. 72, 1536 (1998).

${ }^{23}$ N. Tessler, G. J. Denton, and R. H. Friend, Nature (London) 382, 695 (1996).

${ }^{24}$ M. A. Diaz-Garcia, F. Hide, B. J. Schwartz, M. D. McGehee, M. R. Andersson, and A. J. Heeger, Appl. Phys. Lett. 70, 3191 (1997).

${ }^{25}$ M. D. McGehee and A. J. Heeger, Adv. Mater. (Weinheim, Ger.) 12, 1655 (2000).

${ }^{26}$ V. G. Kozlov and S. R. Forrest, Curr. Opin. Solid State Mater. Sci. 4, 203 (1999).

${ }^{27}$ N. Tessler, Adv. Mater. (Weinheim, Ger.) 11, 363 (1999).

${ }^{28}$ J. Schmidtke, S. Kniesel, and H. Finkelmann, Macromolecules 38, 1357. (2005). 